Pacific Journal of Mathematics

POINT SEPARATION BY BOUNDED ANALYTIC FUNCTIONS
OF A COVERING RIEMANN SURFACE 


\title{
POINT SEPARATION \\ BY BOUNDED ANALYTIC FUNCTIONS OF A COVERING RIEMANN SURFACE
}

\section{Mikihiro Hayashi aNd Mitsuru NAKai}

\begin{abstract}
Results of both positive and negative directions on the point separation by bounded analytic functions of two-sheeted covering Riemann surfaces are given when the points of base Riemann surfaces are separated by bounded analytic functions.
\end{abstract}

1. Introduction. Let $R$ be a Riemann surface and $H^{\infty}(R)$ the set of bounded analytic functions on $R$. In the study of bounded analytic functions on Riemann surfaces, one of the basic problems is to determine when the points of $R$ are separated by $H^{\infty}(R)$. Here we say that $H^{\infty}(R)$ separates the points of $R$ (or $H^{\infty}(R)$ is separating) if for any pair of distinct points $a$ and $b$ of $R$ there exists an $f$ in $H^{\infty}(R)$ with $f(a) \neq f(b)$. Although we do not have any satisfactory answer to the problem, there is a very general result on the point separation by an algebra of analytic functions by Royden [5]. Applied to the present case the Royden result amounts to saying that if a Riemann surface $\tilde{R}$ admits a nonconstant bounded analytic function, then there is a quotient Riemann sueface $R$ of $\tilde{R}$ with a quotient map $\psi$ of $\tilde{R}$ onto $R$ such that $H^{\infty}(R)$ is isomorphic to $H^{\infty}(\tilde{R})$ via the correspondence $f \rightarrow f \circ \psi$ and such that $H^{\infty}(R)$ is weakly separating, by which we mean that $H^{\infty}(R)$ separates the points of $R$ except for a countable subset of $R$. At present, the gap between this general result of Royden and our knowledge on concrete examples is wide. For this reason it might be natural to consider the problem in the following special case as an experimental study.

Suppose there is given a Riemann surface $R$ such that $H^{\infty}(R)$ is separating. For a Riemann surface $\tilde{R}$ with a holomorphic proper mapping $\psi$ of $\tilde{R}$ onto $R$ (i.e., $\tilde{R}$ is a ramified finitely sheeted unlimited covering surface of $R)$, we ask when $H^{\infty}(\tilde{R})$ is separating.

The problem has been considered by Selberg [8], and later by Stanton [9] when the base domain $R$ is the open unit disk, and then by Segawa [7] when $R$ is a Riemann surface of Parreau-Widom type. In this note, we continue to study the problem in the case when the base 
Riemann surface is rather more general. In this context we also cite here a related work of Forelli [1]. The contents of our paper are as follows.

In $\S 2$, we consider as $\tilde{R}$ a subdomain of the famous Myrberg surface (cf. e.g. [6]) which is obtained by deleting a sequence of disjoint disks around ramification points. We shall prove that $H^{\infty}(\tilde{R})$ for this $u n$ ramified $\tilde{R}$ is not separating if the radii of disks are chosen to decrease rapidly enough, and also that $H^{\infty}(\tilde{R})$ is separating if they are chosen to decrease slowly enough. In spite of the fact that these results are likely to happen, our proof of the "not separating" case, in particular, will need some elaborate arguments.

In $\S 3$, we examine the following conjecture.

Conjecture 1.1. Suppose that $H^{\infty}(R)$ is separating. Consider two copies of $R \backslash J$, where $J$ is the union of a finite number of mutually disjoint compact slits on $R$. Let $\tilde{R}$ be the Riemann surface obtained by joining one copy to the other along the two sides of the corresponding slits crosswise. Then, $H^{\infty}(\tilde{R})$ is separating.

We shall show that the conjecture is true if, in addition, the slits are chosen in a certain open subset of $R$, and that the conjecture fails, surprisingly, in general. In fact, an example of a Riemann surface $R$ constructed in [4] will show that the conjecture is true if the slits sit in a certain place of $R$, but it fails if the slits sit in some other place of the same $R$.

In this note, we only consider the two-sheeted covering case. Thus, our results are by no means complete, and there are more problems left than answers given in this paper.

2. We denote by $\Delta(x, r)(\bar{\Delta}(x, r)$, resp.) an open (closed, resp.) disk in the complex plane $C$ with center $x$ and radius $r$. Let $\Delta_{0}=\Delta(0,1) \backslash\{0\}$ and $\Delta_{k}=\bar{\Delta}\left(2^{-k}, r_{k}\right), k=1,2,3, \ldots$ We assume that the closed disks $\Delta_{k}$ are mutually disjoint and included in $\Delta_{0}$, that is, $2^{-k-1}+r_{k+1}<$ $2^{-k}-r_{k}$. Put

$$
R=\Delta_{0} \backslash \bigcup_{k=1}^{\infty} \Delta_{k} .
$$

Let $\tilde{\Delta}_{0}$ be the two-sheeted unlimited covering surface of $\Delta_{0}$ whose ramification points are those over $z=2^{-k}$ for $k=1,2,3, \ldots$, and let $\psi$ denote the covering map of $\tilde{\Delta}_{0}$ onto $\Delta_{0}$. Define $\tilde{R}=\psi^{-1}(R)$. Then, the Riemann surface $\tilde{R}$ is a two-sheeted smooth covering of the domain $R$. Now we show the following. 
THEOREM 2.1. Suppose, with the above notation, that the origin is an irregular boundary point for the domain $\Delta_{0}$ in the sense of the potential theory, i.e.,

$$
\sum_{k=1}^{\infty} \frac{k}{\log \left(1 / r_{k}\right)}<\infty
$$

Then, $H^{\infty}(\tilde{R})$ does not separate the points of any fibre $\psi^{-1}(z)$ for any $z \in R$. Thus, $H^{\infty}(\tilde{R})=H^{\infty}(R) \circ \psi$.

We need the following lemma.

LEMMA 2.2. Let $u(z)$ be a bounded real-valued harmonic function defined in an annulus $\{z: a<|z|<b\}$. Then,

$$
\sup _{\theta, \phi \in[0,2 \pi]}\left|u\left(\sqrt{a b} e^{i \theta}\right)-u\left(\sqrt{a b} e^{i \phi}\right)\right| \leq \frac{24 \sqrt{a / b}}{(1-\sqrt{a / b})^{2}}\|u\|_{\infty} .
$$

Proof. Since the estimate is invariant under $z \rightarrow z / b$, we may assume that $b=1$. We may also assume that $u$ is continuous on $a \leq|z| \leq b$. First, we let $-1 \leq u(\zeta) \leq 1$ for $|\zeta|=1$ and $u(\zeta)=0$ for $|\zeta|=a$. We consider the Poisson integral

$$
v\left(\mathrm{re}^{i \phi}\right)=\int_{0}^{2 \pi} \frac{1-r^{2}}{1-2 r \cos (\theta-\phi)+r^{2}} u\left(e^{i \theta}\right) d \theta / 2 \pi
$$

on the unit disk. Then,

$$
\left|v\left(\mathrm{re}^{i \phi}\right)-v(0)\right| \leq \int_{0}^{2 \pi} \frac{2 r+2 r^{2}}{1-2 r+r^{2}} d \theta / 2 \pi=\frac{4 r}{(1-r)^{2}} .
$$

Put $w=u-v$. Then, $w(\zeta)=0$ for $|\zeta|=1$, and $w(\zeta)=-v(\zeta)$ for $|\zeta|=a$. Hence,

$$
|w(\zeta)+v(0)| \leq 4 a /(1-a)^{2}
$$

for $|\zeta|=a$. By the maximum principle, it follows that

$$
\left|w(z)+v(0) \frac{\log |z|}{\log a}\right| \leq \frac{4 a \log |z|}{(1-a)^{2} \log a} .
$$

From this inequality, we see

$$
\left|w\left(\sqrt{a} e^{i \theta}\right)-w\left(\sqrt{a} e^{i \phi}\right)\right| \leq 4 a /(1-a)^{2} .
$$

Also, by (2.2), we have

$$
\left|v\left(\sqrt{a} e^{i \theta}\right)-v\left(\sqrt{a} e^{i \phi}\right)\right| \leq 8 \sqrt{a} /(1-\sqrt{a})^{2} .
$$


The last two inequalities imply

$$
\left|u\left(\sqrt{a} e^{i \theta}\right)-u\left(\sqrt{a} e^{i \phi}\right)\right| \leq 12 \sqrt{a} /(1-\sqrt{a})^{2} .
$$

For a general $u$, we write $u=u_{1}+u_{2}$, where $u_{1}$ and $u_{2}$ are harmonic with boundary values $u_{1}\left(e^{i \theta}\right)=u\left(e^{i \theta}\right)$ and $u_{2}\left(a e^{i \theta}\right)=u\left(a e^{i \theta}\right)$. Applications of the above argument to $u_{1}(z)$ and $u_{2}(a / z)$ imply the required estimate.

Proof of Theorem 2.1. Let $k$ be a positive integer. By (2.1), $k / \log _{2}\left(1 / r_{k}\right)=o(1)$, i.e., if $r_{k}=2^{-n(k) k}$, then $n(k) \rightarrow+\infty$ as $k \rightarrow \infty$. In this proof, we admit a conventional use of the symbol $n(k)$ to represent any sequence of real numbers with the property $n(k) \rightarrow+\infty$ as $k \rightarrow \infty$, that is, $n(k)$ will not be a fixed sequence. For every positive integer $n$, we have

$$
\sum_{k=1}^{\infty} 2^{n k} \sqrt{r_{k}}<\infty ; \quad \text { and }
$$

$$
\sum_{k=1}^{\infty} 2^{n k} r_{k}<\infty
$$

Let $f \in H^{\infty}(R)$. By (2.4), we see that

$$
f^{(n)}(0)=\sum_{k=0}^{\infty} \frac{n !}{2 \pi i} \int_{\partial \Delta_{k}} \frac{f(\zeta)}{\zeta^{n+1}} d \zeta
$$

defines a bounded linear functional on $H^{\infty}(R)$. Here, the directions of integral paths are taken counter-clockwise for $\partial \Delta_{0}$, and clockwise for $\partial \Delta_{k}, k=1,2, \ldots$, so that this formula is obviously true in the classical sense if $f$ is analytic in a neighborhood of the origin. Also, we have

$$
f(z)=\sum_{k=0}^{\infty} \frac{1}{2 \pi i} \int_{\partial \Delta_{k}} \frac{f(\zeta)}{\zeta-z} d \zeta
$$

for $z \in R$.

The theorem follows from the following assertion.

Assertion 2.3. Let $f \in H^{\infty}(R)$.

(i) If $f^{(n)}(0)=0$ for all $n=0,1,2, \ldots$, then $f=0$.

(ii) If there are a sequence $\left\{\rho_{k}\right\}$ of points in $R$ and positive constants $A, A_{n}$ such that $\rho_{k} \rightarrow 0$,

$$
\left|f\left(\rho_{k}\right)\right| \leq A 2^{-n(k) k} \text { and }\left|\rho_{k+i}-\rho_{k+j}\right| \geq A_{n} 2^{-k}
$$

for all $k$ and $i \neq j, 0 \leq i, j \leq n$,

then $f^{(n)}(0)=0$ for all $n=0,1,2, \ldots$ 
In fact, let $g \in H^{\infty}(\tilde{R})$. For each point $z$ in $R$, we write $\psi^{-1}(z)=$ $\left\{z_{+}, z_{-}\right\}$and define

$$
f(z)=\left(g\left(z_{+}\right)-g\left(z_{-}\right)\right)^{2} .
$$

As is well-known, $f(z)$ is a single-valued bounded analytic function on $R$. In order to see the conditions in the hypothesis of the assertion (ii), we consider the annulus $\Delta_{k}^{*}=\left\{z: r_{k}<\left|z-2^{-k}\right|<2^{-k} / 4\right\}$. Note that $\Delta_{k}^{*}$ is included in $R$ for every large $k$ and that $\psi^{-1}\left(\Delta_{k}^{*}\right)$ is conformally equivalent to the annulus $\left\{w: \sqrt{r_{k}}<|w|<2^{-k / 2-1}\right\}$. The conformal radius of $\psi^{-1}\left(\Delta_{k}^{*}\right)$ is estimated as follows:

$$
\sqrt{r_{k}} / 2^{-k / 2-1}=\left(r_{k} 2^{k+2}\right)^{1 / 2} \leq 2^{-n(k) k}
$$

when $k$ is large. Now, put $\rho_{k}=2^{-k}+\sqrt{r_{k}} 2^{-k / 2-1}$. Then, $\mid \rho_{m+i}-$ $\rho_{m+j} \mid \geq 2^{-m} / 2^{n+1}$ and, by Lemma 2.2 ,

$$
\left|f\left(\rho_{k}\right)\right|<2^{-n(k) k}\|g\|_{\infty}^{2} .
$$

Hence, we have $f=0$ by the assertion. It remains to prove the assertion.

Proof of Assertion 2.3. (i) Set $\Delta_{k}^{\prime}=\bar{\Delta}\left(2^{-k}, \sqrt{r_{k}}\right)$. By (2.3), disks $\Delta_{k}^{\prime}$ are mutually disjoint for large $k$. For the simplicity of the argument, we may assume that all $\Delta_{k}^{\prime}$ are mutually disjoint, and we define

$$
Y=\Delta_{0} \backslash \bigcup_{k=1}^{\infty} \Delta_{k}^{\prime}
$$

Suppose $f^{(n)}(0)=0$ for all $n=0,1,2, \ldots$. Assume on the contrary that $f \neq 0$. Multiplying a nonzero constant to $f$, if necessary, we may assume that $|f(z)|<1$. From (2.5) and (2.6), an induction on $n$ yields the identity

$$
f(z)=z^{n} \sum_{k=0}^{\infty} \frac{1}{2 \pi i} \int_{\partial \Delta_{k}} \frac{f(\zeta)}{\zeta^{n}(\zeta-z)} d \zeta .
$$

It follows from (2.3) that

$$
C_{n}=\sup _{z \in Y} \sum_{k=0}^{\infty} \frac{1}{2 \pi} \int_{\partial \Delta_{k}} \frac{1}{\left|\zeta^{n}(\zeta-z)\right|}|d \zeta|<+\infty .
$$

By (2.8), $|f(z)| \leq C_{n}|z|^{n}(n=0,1,2, \ldots)$ for $z \in Y$, or equivalently, $\log |1 / f(z)| \geq n \log |1 / z|-\log C_{n}(n=0,1,2, \ldots)$. Let $u$ be the lower 
envelope of the family $\{s\}$ of nonnegative superharmonic functions $s$ on $Y$ such that the inferior limit of $s(z)$ as $z \in Y$ approaches to $\zeta$ is not less than $\log |1 / \zeta|$ for every $\zeta \in \partial Y \backslash\{0\}$. Then, $u(z)$ is harmonic on $Y$ with boundary values $\log |1 / \zeta|$ on $\partial Y \backslash\{0\}$. By the condition (2.1), $z=0$ is the irregular boundary point of $Y$ in the sense of potential theory. Hence, $v(z)=\log |1 / z|-u(z)>0$ on $Y$, which can be seen, for instance, by an application of Fatou's lemma to the formula [10; Theorem III.41]. Since $u(z)>0, \log |1 / z|>v(z)$ and we have

$$
\log \frac{1}{|f(z)|} \geq n v(z)-\log C_{n} \quad(n=0,1,2, \ldots)
$$

on $\bar{Y} \backslash\{0\}$. Fix an arbitrary positive number $\varepsilon$ and consider the superharmonic function

$$
s_{n, \varepsilon}(z)=\log \frac{1}{|f(z)|}+\varepsilon \log \frac{1}{|z|}-n v(z)
$$

on $\bar{Y} \backslash\{0\}$. By (2.9) and the effect of the term $\varepsilon \log |1 / z|$, we see that $\lim _{z \in Y, z \rightarrow 0} s_{n, \varepsilon}(z)=+\infty$. Since $|f(z)|<1$ and $v(z)=0$ on $\partial Y \backslash\{0\}$, we conclude that

$$
\liminf _{z \in Y, z \rightarrow \zeta} s_{n, \varepsilon}(z) \geq 0
$$

for every $\zeta \in \partial Y$. The maximum principle for superharmonic functions assures that $s_{n, \varepsilon}(z) \geq 0$ on $Y$. Letting $\varepsilon \downarrow 0$, we have

$$
\log \frac{1}{|f(z)|} \geq n v(z) \quad(n=0,1,2, \ldots)
$$

on $Y$, a contradiction.

(ii) Define

$$
\left(R_{n} f\right)(z)=f(z)-\sum_{l=0}^{n} \frac{f^{(l)}(0)}{l !} z^{l}
$$

Then,

$$
\left(R_{n} f\right)\left[\rho_{m+n}, \ldots, \rho_{m}\right]=f\left[\rho_{m+n}, \ldots, \rho_{m}\right]-f^{(n)}(0) / n !,
$$

where

$$
\begin{aligned}
& F\left[x_{n}, \ldots, x_{1}, x_{0}\right] \\
& \quad=\sum_{k=0}^{n} \frac{F\left(x_{k}\right)}{\left(x_{k}-x_{n}\right) \cdots\left(x_{k}-x_{k+1}\right)\left(x_{k}-x_{k-1}\right) \cdots\left(x_{k}-x_{0}\right)} .
\end{aligned}
$$

It follows from (2.7) that $f\left[\rho_{m+n}, \ldots, \rho_{m}\right]$ tends to zero as $m \rightarrow \infty$. On the other hand, we estimate $\left(R_{n} f\right)\left[\rho_{m+n}, \ldots, \rho_{m}\right]$ as follows. By 
$(2.8)$,

$$
\left(R_{n} f\right)(z)=z^{n+1} \sum_{k=0}^{\infty} \frac{1}{2 \pi i} \int_{\partial \Delta_{k}} \frac{\left(R_{n} f\right)(\zeta)}{\zeta^{n+1}(\zeta-z)} d \zeta
$$

Now, if $\zeta \in \partial \Delta_{k}$, then

$$
\left|\frac{\left(R_{n} f\right)(\zeta)}{\zeta^{n+1}\left(\zeta-\rho_{k}\right)}\right| \leq\left\|R_{n} f\right\|_{\infty} /\left(2^{-k}-r_{k}\right)^{n+1}\left(\sqrt{r_{k}} 2^{-k / 2-1}-r_{k}\right)
$$

and if $\zeta \in \partial \Delta_{l}, l \neq k$, then

$$
\left|\frac{\left(R_{n} f\right)(\zeta)}{\zeta^{n+1}\left(\zeta-\rho_{k}\right)}\right| \leq\left\|R_{n} f\right\|_{\infty} /\left(2^{-l}-r_{l}\right)^{n+1} 2^{-l / 4} .
$$

Hence, by Lebesgue's dominated covergence theorem, we conclude

$$
\lim _{m \rightarrow \infty}\left(R_{n} f\right)\left[\rho_{m+n}, \ldots, \rho_{m}\right]=0 .
$$

This shows that $f^{(n)}(0)=0$ for all $n$, as was to be proved.

One can also prove (2.10) using an estimate in terms of analytic capacity [3: Estimate $\left.2.7\left(\mathrm{E}-3^{\prime}\right)\right]$.

THEOREM 2.4. Suppose that, with the same notation as above, we have inf $r_{k} 2^{k}>0$. Then, $H^{\infty}(\tilde{R})$ separates the points of $\tilde{R}$.

Proof. Set $\delta=\inf _{k} r_{k} 2^{k}$, which is seen to be less than $1 / 3$. If we replace $r_{k}$ by $\delta 2^{-k}$, then $\tilde{R}$ is replaced by a larger subdomain of $\tilde{\Delta}_{0}$. Hence, we may assume that $r_{k}=\delta 2^{-k}$. Set $g_{n}(z)=z /\left(z-2^{-n}\right)$ for $n=1,2,3, \ldots$. The following inequalities are immediate:

$$
\begin{gathered}
\left|1-g_{n}(z)\right| \leq 2^{-n+1} /|z|, \quad|z| \geq 2^{-n+1}, \\
\left|g_{n}(z)\right| \leq 2^{-n+1} / r_{n}=2 / \delta, \quad\left|z-2^{-n}\right| \geq r_{n}, \\
\left|g_{n}(z)\right| \leq 1, \quad \operatorname{Re} z \leq 2^{-n-1}, \\
\left|g_{n}(z)\right| \leq 1+4 \cdot 2^{k-n}, \quad\left|z-2^{-k}\right|=r_{k}, \quad k \leq n-2 .
\end{gathered}
$$

Here, (2.14) follows from (2.11). By estimate (2.11), the function

$$
g=\prod_{n=1}^{\infty} g_{n}
$$

converges almost uniformly on $\mathbf{C} \backslash\{0\}$. Regarding the function $g$ as a product of functions $g_{2 n-1} g_{2 n}$, we see that the square root $f=\sqrt{g}$ 
defines a single-valued meromorphic function on the covering surface $\tilde{\Delta}_{0}$ of $\Delta_{0}$. By $(2.12),(2.13)$ and $(2.14)$, we have

$$
\left|\prod_{n=1}^{m} g_{n}\right| \leq \frac{2}{\delta} \prod_{n=k+2}^{m}\left(1+4 / 2^{n-k}\right)
$$

on $\left|z-2^{-k}\right|=r_{k}$ for any integer $m \geq k+2$. Hence,

$$
\left|\prod_{n=1}^{m} g_{n}\right| \leq \frac{2}{\delta} \prod_{n=1}^{\infty}\left(1+4 / 2^{n+1}\right)
$$

on $R$ for any positive integer $m$. This implies that the function $g$ is bounded on $R$, and hence, $f$ is a bounded analytic function on $\tilde{R}$. Clearly, the function $f$ separates the fibre $\psi^{-1}(z)$ for every $z \in R$.

In the above notations, it is an interesting unsettled question to find a necessary and sufficient condition on radii $r_{k}$ in order that $H^{\infty}(\tilde{R})$ is separating.

3. In this section an arbitrary open Riemann surface $R$ will be considered. We note by $M^{\infty}(R)$ the set of meromorphic functions on $R$ bounded off compact subsets of $R$ and by $\mathscr{P}(R)$ the set of points $p$ of $R$ such that there exists an $f$ in $M^{\infty}(R)$ for which $p$ is a pole. The set $\mathscr{P}(R)$, referred to as the pole set of $R$, is seen to be open by considering $1 /(f-\alpha)$ for $f$ in $M^{\infty}(R)$ and $\alpha$ complex numbers with large absolute values. In the proof of the following theorem, a Cauchy differential $A(\zeta, z) d \zeta((\zeta, z) \in \mathscr{P}(R) \times R)$ constructed in [2] under the assumption that $H^{\infty}(R)$ is weakly separating will be used essentially. Let $V$ be an arbitrary parametric disk in $\mathscr{P}(R)$. The coefficients $A(\zeta, z)$ $((\zeta, z) \in V \times R)$ of the above differential enjoy the following properties: $(\alpha) A(\zeta, z)$ is holomorphic on $V \times R$ except for the set $\zeta=z,(\beta)$ $A(\zeta, z)=1 /(\zeta-z)+$ (holomorphic function) on $V \times V,(\gamma) A(\zeta, \cdot)$ is bounded analytic on $R \backslash V$ for any fixed $\zeta$ in $V$, and $(\delta) A\left(\zeta_{n}, \cdot\right) \rightarrow$ $A(\zeta, \cdot)$ uniformly on $R \backslash V$ as $\zeta_{n} \rightarrow \zeta$ in $V$.

We have the following.

THeOREM 3.1. Let $\psi$ be a two-sheeted unlimited covering map of a Riemann surface $\tilde{R}$ onto a Riemann surface $R$. Suppose that $H^{\infty}(R)$ is (weakly) separating and that there exists an open subset $W$ of $R$ with 
the following properties:

(3.1) $\quad H^{\infty}(\tilde{W})$ is (weakly) separating, where $\tilde{W}=\psi^{-1}(W)$;

(3.2) the boundary $\partial W$ of $W$ is a compact subset

of the pole set $\mathscr{P}(R) ;$ and

(3.3) $\quad \psi^{-1}(R \backslash W)$ splits into two disjoint copies of $R \backslash W$.

Then, $H^{\infty}(\tilde{R})$ is (weakly) separating on $\tilde{R}$.

Before proceeding to the proof of the above, we consider some examples. Let $J_{1}, J_{2}, \ldots, J_{n}$ be a finite number of mutually disjoint compact analytic Jordan arcs contained in the set $\mathscr{P}(R)$. Let $R_{+}$and $R_{-}$be two copies of $R \backslash\left(\bigcup_{k} J_{k}\right)$, and let $\tilde{R}$ be the Riemann surface obtained by joining $R_{+}$with $R_{-}$along two sides of each $J_{k}$ crosswise. Choosing a disjoint union of disks containing $J_{k}$ as the open subset $W$, we see that conditions (3.1), (3.2) and (3.3) are satisfied. Therefore Conjecture 1.1 is true in this case. Furthermore, we can apply the theorem to the case when $\left\{J_{k}\right\}$ is an infinite sequence if $J_{k}$ are suitably chosen (see remarks after the following example).

EXAMPLE 3.2. Conjecture 1.1 fails in general. More precisely, let $J$ be a compact slit in $R$ and $\tilde{R}$ the two-sheeted covering Riemann surface obtained by joining two copies of $R \backslash J$ along $J$ crosswise. Then, there exists a Riemann surface $R$ such that $H^{\infty}(R)$ is separating, but $H^{\infty}(\tilde{R})$ may or may not be separating, depending upon the choice of the slit $J$ in $R$.

Proof. We recall an example of a Riemann surface $R$ constructed in [4]. Namely, let $I_{1}, I_{2}, \ldots$ be disjoint closed intervals on the interval $[0,1)$ such that $I_{k}$ converges to $z=1$. We consider a union $I_{k}^{*}$ of a finite number of disjoint subintervals of $I_{k}$ for each $k=1,2, \ldots$. Let $R_{0}=\Delta \backslash\left(\bigcup_{n=1}^{\infty} I_{n}^{*}\right)$ and $R_{k}=\Delta \backslash\left\{I_{k}^{*} \cup\left(\bigcup_{j=1}^{k-1} I_{j}\right)\right\}$, where $\Delta$ is the open unit disk. The Riemann surface $R$ is now obtained by joining every $R_{k}$ with $R_{0}$ along every subinterval contained in $I_{k}^{*}$ crosswise. If one chooses $I_{k}^{*}$ in such a fashion that it includes sufficiently many subintervals, then $f \mid R_{k}$ converges to $f \mid R_{0}$ almost uniformly for every bounded analytic function $f$ on $R$. Also, we know that $H^{\infty}(R)$ is separating and that $\mathscr{P}(R)=\bigcup_{k=1}^{\infty} R_{k}$ (cf. [4] for details). If we choose the slit $J$ in $\mathscr{P}(R)$, then $H^{\infty}(\tilde{R})$ is separating by the theorem. Next, we choose the slit $J$ in the bottom sheet $R_{0}$. Let $\psi$ be the covering map of $\tilde{R}$ onto $R$. Consider a closed Jordan curve $C$ on $R_{0}$ enclosing $J$. 
Choosing the union $I_{k}^{*}$ of subintervals more carefully, if necessary, we can assume that $g \mid R_{k}$ converges uniformly to $g \mid\left(R_{0} \backslash J\right)$ on $C$ for every bounded analytic function $g$ on $R \backslash J$, so that $g$ extends analytically to $R$. Now, let $f$ be a bounded analytic function on $\tilde{R}$ and let $R_{+}$be one of two connected components of $\psi^{-1}(R \backslash J)$. Since $\psi$ is one-toone on the domain $R_{+}, f \circ \psi^{-1}$ defines a function $g$ on $R \backslash J$. Hence, $g$ extends analytically to $R$. Since the analytic function $g \circ \psi$ on $\tilde{R}$ agrees with $f$ on $R_{+}$, we have $g \circ \psi=f$ on $\tilde{R}$ throughout. This shows that $H^{\infty}(\tilde{R})$ does not separate any fibre $\psi^{-1}(p)$ for any $p \in R$.

Here are two remarks on the above example. First, suppose we construct a Riemann surface $S$ by joining $R \backslash J$ with $\Delta \backslash J$ along $J$ crosswise. Namely, we attach an open disk to the bottom sheet of $R$. In this case, applying a similar argument, we easily see not only the fact that $H^{\infty}(S)$ is not separating but also the fact that $H^{\infty}(S)$ turns back to the algebra $H^{\infty}(\Delta)$ on the unit disk $\Delta$. That is, $H^{\infty}(S)$ does not separate any pair of distinct points in the fibre $\phi^{-1}(z)$ for every $z \in \Delta$, where $\phi$ is a covering map of $S$ onto the open unit disk $\Delta$.

Second, suppose we choose an infinite number of mutually disjoint slits $J_{k}$ in a fixed sheet (or finite number of sheets) $R_{n}(n \geq 1)$ of $R$ such that $\sum\left(1-\left|a_{k}\right|\right)<\infty$, where $a_{2 k-1}$ and $a_{2 k}$ are the end points of the slit $J_{k}$. If we construct a two-sheeted covering $\tilde{R}$ of $R$ as before, then Theorem 3.1 shows that $H^{\infty}(\tilde{R})$ is separating.

The following lemma will be needed in the proof of Theorem 3.1.

LEMMA 3.3. Let $\Gamma$ be a union of a finite number of disjoint closed Jordan curves contained in $\mathscr{P}(R)$, and let $g$ be an analytic function defined on a neighborhood of $\Gamma$. If $g$ has no zeros on $\Gamma$, then there exists a function $h$ in $M^{\infty}(R)$ such that $\log (h g)$ has a single-valued analytic branch on a neighborhood of $\Gamma$.

Proof. Let $\Gamma=\Gamma_{1} \cup \cdots \cup \Gamma_{p}$, where $\Gamma_{j}$ are disjoint closed Jordan curves. For each $\Gamma_{j}$, we can choose an annular neighborhood $V_{j}$ of it and a conformal analtyic map $h_{j}$ of $V_{j}$ onto an annulus $\{w: \delta<|w|<$ $1 / \delta\}(0<\delta<1)$. We may further assume that $V_{j}$ 's are mutually disjoint. Note that the period of $\log g$ along the curve $\Gamma_{j}$ is an integer multiple of $2 \pi i$, say $2 \pi i m_{j}$. Shrinking $V_{j}$, if necessary, we see that $\log g / h_{j}^{m_{j}}$ has a single-valued analytic branch on $V_{j}$. Since $V_{j}$ 's are mutually disjoint, by using the Cauchy differential $A(\zeta, z)$ introduced in the beginning of this section, a Runge type approximation gives us a meromorphic function $h$ in $M^{\infty}(R)$ such that $\left|1 / h_{j}^{m_{j}}-h\right|<\delta^{m_{j}}$ on $\Gamma_{j}$. 
Then, $\left|1-h_{j}^{m_{j}} h\right|<1$ on $\Gamma_{j}$, and hence, $\log \left(h_{j}^{m_{\jmath}} h\right)$ has a single-valued analytic branch on a neighborhood of $\Gamma_{j}$. Consequently,

$$
\log (h g)=\log \left(h_{j}^{m_{j}} h\right)+\log \left(g / h_{j}^{m_{j}}\right)
$$

has a single-valued analytic branch on a neighborhood of $\Gamma_{j}$. This proves the lemma.

Proof of Theorem 3.1. Set $\Gamma=\partial W$ and consider a neighborhood $V$ of $\Gamma$ such that $\bar{V} \subset \mathscr{P}(R)$ and such that the boundary $\partial V$ of $V$ consists of a finite number of disjoint closed Jordan curves. By condition (3.3), there are no ramification points over $\Gamma$. Hence, we may further assume that $\tilde{V}=\psi^{-1}(V)$ consists of two disjoint homeomorphic copies $V^{+}$and $V^{-}$of $V$. Now we see that all the hypotheses remain valid even when one replaces the open set $W$ by a slightly smaller one. Therefore, we may assume, from the beginning, that $\Gamma$ consists of a finite number of disjoint closed Jordan curves, and also, that the neighborhood $V$ is chosen as a disjoint union of annular neighborhoods which include each one of the components of $\Gamma$. Let $\left\{R_{j}\right\}_{j=1}^{p}$ be the connected components of $R \backslash W$ and set $\Gamma_{j}=\partial R_{j}$, where each $\Gamma_{j}$ may consist of a finite number of disjoint closed Jordan curves. Denote by $V_{j}$ the union of the annular components of $V$ containing a component of $\Gamma_{j}$. Set

$$
W_{0}=W \cup V \text { and } W_{j}=R_{j} \cup V_{j} \text { for } j=1, \ldots, p \text {. }
$$

Shrinking $W$ and $V$, if necessary, we may assume by condition (3.1) that $H^{\infty}\left(\tilde{W}_{0}\right)$ is (weakly) separating, where $\tilde{W}_{0}=\psi^{-1}\left(W_{0}\right)$.

Now, if $z \in R$ is not a ramification point, then the point $z$ has two pre-images, i.e., $\psi^{-1}(z)=\left\{z^{+}, z^{-}\right\}$. Define $\tau\left(z^{+}\right)=z^{-}$and $\tau\left(z^{-}\right)=$ $z^{+}$for every such $z$. As is well-known, $\tau$ extends to a conformal mapping of $\tilde{R}$ onto itself and $\tau \circ \tau=$ identity. Note that $f \circ \psi \in H^{\infty}(\tilde{R})$ for every $f \in H^{\infty}(R)$. Hence, in order to see that $H^{\infty}(\tilde{R})$ is weakly separating, it suffices to find a function $F$ in $H^{\infty}(\tilde{R})$ with $F \circ \tau \neq F$. Since $H^{\infty}\left(\tilde{W}_{0}\right)$ is (weakly) separating, there is a function $G$ in $H^{\infty}\left(\tilde{W}_{0}\right)$ such that $G \circ \tau \neq G$. Replacing $G$ by $G-G \circ \tau$, we assume that

$$
G \circ \tau=-G
$$

and $G$ is not identically zero. Also, we may assume that $G(\tilde{z})$ has no zeros on $\tilde{V}=\psi^{-1}(V)$, for one may modify, if necessary, the boundary $\Gamma$ of $W$ slightly, and replace the neighborhood $V$ of $\Gamma$ by a smaller one. Let us regard the restriction of $G$ to $V^{+}$as a function on $V$, which we denote by $g$. By Lemma 3.3, there is a function $h$ in $M^{\infty}(R)$ such 
that $\log (g h)$ is single-valued and analytic in $V$, where we replace $V$ by a smaller one if necessary, again. Set $u=\log (g h)$. Let $A(\zeta, z) d \zeta$ be the Cauchy differential on $\mathscr{P}(R) \times R$ introduced in the beginning of this section. For $z \in V_{j}$, it follows that

$$
u(z)=\frac{1}{2 \pi i} \int_{\Gamma_{,}^{\prime \prime}} u(\zeta) A(\zeta, z) d \zeta-\frac{1}{2 \pi i} \int_{\Gamma_{j}^{\prime}} u(\zeta) A(\zeta, z) d \zeta
$$

where $\Gamma_{j}^{\prime}=W \cap \partial V_{j}$ and $\Gamma_{j}^{\prime \prime}=R_{j} \cap \partial V_{j}$. Define

$$
\begin{aligned}
u_{0}(z)= & \frac{1}{2 \pi i} \sum_{j} \int_{\Gamma_{j}^{\prime \prime}} u(\zeta) A(\zeta, z) d \zeta, \quad z \in W_{0}, \\
u_{j}(z)= & \frac{1}{2 \pi i} \sum_{k \neq j} \int_{\Gamma_{k}^{\prime \prime}} u(\zeta) A(\zeta, z) d \zeta \\
& +\frac{1}{2 \pi i} \int_{\Gamma_{j}^{\prime}} u(\zeta) A(\zeta, z) d \zeta, \quad z \in W_{j}
\end{aligned}
$$

for $j=1, \ldots, p$. Note that

(3.5) $\quad u_{0}$ is a bounded analytic function on $W_{0} \backslash V$ and $u_{j}$ is a bounded analytic function on $W_{j} \backslash V$.

Furthermore, we have

$$
u_{j}=u_{0}-u, \quad z \in V_{j} .
$$

By condition (3.3), $\psi^{-1}\left(W_{j}\right)$ consists of two homeomorphic copies $W_{j}^{+}$and $W_{j}^{-}$of $W_{j}$, where $W_{j}^{+}$and $W_{j}^{-}$intersect with $V^{+}$and $V^{-}$, respectively. We now define a meromorphic function $F$ on $\tilde{R}$ by

$$
F(\tilde{z})= \begin{cases}\exp \left(-u_{j} \circ \psi(\tilde{z})\right), & \tilde{z} \in W_{j}^{+}, \\ -\exp \left(-u_{j} \circ \psi(\tilde{z})\right), & \tilde{z} \in W_{j}^{-}, \\ G(\tilde{z})(h \circ \psi)(\tilde{z}) \exp \left(-u_{0} \circ \psi(\tilde{z})\right), & \tilde{z} \in \tilde{W}_{0} .\end{cases}
$$

By (3.4) and (3.6), $F(\tilde{z})$ is well-defined. It follows that $F \in M^{\infty}(\tilde{R})$ and that $F \circ \tau=-F$. By [2], we can find a nonzero bounded analytic function $f$ on $R$ such that $f h \in H^{\infty}(R)$. Hence, $(f \circ \psi) F \in H^{\infty}(\tilde{R})$. Therefore, $H^{\infty}(\tilde{R})$ is weakly separating. If $H^{\infty}(\tilde{W})$ is separating, we can choose in the foregoing discussion a function $G$ and a function $f$ for each $z \in W$ such that $G\left(z^{+}\right)=-G\left(z^{-}\right) \neq 0$ and $(f h)(z) \neq 0$. Hence, the function of the form $(f \circ \psi) F$ separates the points $z^{+}$and $z^{-}$for each $z \in W$. The same is true for $z \in R \backslash W$, because $F$ does not vanish on $\tilde{R} \backslash \tilde{W}$. This proves that $H^{\infty}(\tilde{R})$ is separating. 


\section{REFERENCES}

[1] F. Forelli, A note on divisibility in $H^{\infty}(X)$, Canad. J. Math., 36 (1984), 458-469.

[2] T. W. Gamelin and M. Hayashi, The algebra of the bounded analytic functions on a Riemann surface, J. Reine Angew. Math., 382 (1987), 49-73.

[3] M. Hayashi, Smoothness of analytic functions at boundary points, Pacific J. Math., 67 (1976), 171-202.

[4] _ The maximal ideal space of the bounded analytic functions on a Riemann surface, J. Math. Soc. Japan, 39 (1987), 337-344.

[5] H. L. Royden, Algebras of bounded analytic functions on Riemann surfaces, Acta Math., 114 (1965), 113-142.

[6] L. Sario and M. Nakai, Classification Theory of Riemann Surfaces, SpringerVerlag, 1970.

[7] S. Segawa, Bounded analytic functions on unbounded covering surfaces, Pacific J. Math., 79 (1978), 183-187.

[8] H. L. Selberg, Ein Satz über beschränkte endlichvieldeutige analytische Funktionen, Comm. Math. Helv., 9 (1937), 104-108.

[9] C. M. Stanton, Bounded analytic functions on a class of Riemann surfaces, Pacific J. Math., 59 (1975), 557-565.

[10] M. Tsuji, Potential Theory in Modern Function Theory. 2nd. ed., Chelsea, New York, 1975.

Received February 20, 1987. To complete the present work the first (second, resp.) named author was supported in part by Grant-in-Aid for Scientific Research No. 60302004,61540071 (61540094, resp.), Japanese Ministry of Education, Science and Culture.

HOKKAIDO UNIVERSITY

SAPPORO 060

JAPAN

AND

Nagoya Institute of Technology

Gokiso, Showa, Nagoya 466

JAPAN 



\section{EDITORS}

\author{
V. S. VARADARAJAN \\ (Managing Editor) \\ University of California \\ Los Angeles, CA 90024 \\ HeRBert Clemens \\ University of Utah \\ Salt Lake City, UT 84112 \\ R. FINN \\ Stanford University \\ Stanford, CA 94305
}

\author{
HERMANN FLASCHKA \\ University of Arizona \\ Tucson, AZ 85721
}

RAMESh A. GANGOLLI University of Washington Seattle, WA 98195

VAUGHAN F. R. JONES University of California

Berkeley, CA 94720
ROBION KIRBY

University of California

Berkeley, CA 94720

C. C. MOORE

University of California

Berkeley, CA 94720

HAROLD STARK

University of California, San Diego

La Jolla, CA 92093

\section{ASSOCIATE EDITORS}
R. ARENS
E. F. BECKENBACH
B. H. NEUMANN
F. WOLF
K. YOSHIDA

(1906-1982)

\section{SUPPORTING INSTITUTIONS}

UNIVERSITY OF ARIZONA
UNIVERSITY OF BRITISH COLUMBIA
CALIFORNIA INSTITUTE OF TECHNOLOGY
UNIVERSITY OF CALIFORNIA
MONTANA STATE UNIVERSITY
UNIVERSITY OF NEVADA, RENO
NEW MEXICO STATE UNIVERSITY
OREGON STATE UNIVERSITY

UNIVERSITY OF ARIZONA

UNIVERSITY OF BRITISH COLUMBIA

UNIVERSITY OF CALIFORNIA

MONTANA STATE UNIVERSITY

NEW MEXICO STATE UNIVERSITY

OREGON STATE UNIVERSITY

\author{
UNIVERSITY OF OREGON \\ UNIVERSITY OF SOUTHERN CALIFORNIA \\ STANFORD UNIVERSITY \\ UNIVERSITY OF HAWAII \\ UNIVERSITY OF TOKYO \\ UNIVERSITY OF UTAH \\ WASHINGTON STATE UNIVERSITY \\ UNIVERSITY OF WASHINGTON
}

The Supporting Institutions listed above contribute to the cost of publication of this Journal, but they are not owners or publishers and have no responsibility for its content or policies.

Mathematical papers intended for publication in the Pacific Journal of Mathematics should be in typed form or offset-reproduced (not dittoed), double spaced with large margins. Please do not use built up fractions in the text of the manuscript. However, you may use them in the displayed equations. Underline Greek letters in red, German in green, and script in blue. The first paragraph must be capable of being used separately as a synopsis of the entire paper. In particular it should contain no bibliographic references. Please propose a heading for the odd numbered pages of less than 35 characters. Manuscripts, in triplicate, may be sent to any one of the editors. Please classify according to the scheme of Math. Reviews, Index to Vol. 39. Supply name and address of author to whom proofs should be sent. All other communications should be addressed to the managing editor, or Elaine Barth, University of California, Los Angeles, California 90024.

There are page-charges associated with articles appearing in the Pacific Journal of Mathematics. These charges are expected to be paid by the author's University, Government Agency or Company. If the author or authors do not have access to such Institutional support these charges are waived. Single authors will receive 50 free reprints; joint authors will receive a total of 100 free reprints. Additional copies may be obtained at cost in multiples of 50 .

The Pacific Journal of Mathematics is issued monthly as of January 1966. Regular subscription rate: $\$ 190.00$ a year (5 Vols., 10 issues). Special rate: $\$ 95.00$ a year to individual members of supporting institutions.

Subscriptions, orders for numbers issued in the last three calendar years, and changes of address should be sent to Pacific Journal of Mathematics, P.O. Box 969, Carmel Valley, CA 93924, U.S.A. Old back numbers obtainable from Kraus Periodicals Co., Route 100, Millwood, NY 10546.

The Pacific Journal of Mathematics at P.O. Box 969, Carmel Valley, CA 93924 (ISSN 0030-8730) publishes 5 volumes per year. Application to mail at Second-class postage rates is pending at Carmel Valley, California, and additional mailing offices. Postmaster: send address changes to Pacific Journal of Mathematics, P.O. Box 969, Carmel Valley, CA 93924.

\section{PUBLISHED BY PACIFIC JOURNAL OF MATHEMATICS, A NON-PROFIT CORPORATION} Copyright (c) 1988 by Pacific Journal of Mathematics 


\section{Pacific Journal of Mathematics}

Vol. 134, No. $2 \quad$ June, 1988

P. D. Allenby and M. Sears, Extension of flows via discontinuous functions

Arthur William Apter and Moti Gitik, Some results on Specker's

problem

Shiu-Yuen Cheng and Johan Tysk, An index characterization of the catenoid and index bounds for minimal surfaces in $\mathbf{R}^{4}$

Mikihiro Hayashi and Mitsuru Nakai, Point separation by bounded analytic functions of a covering Riemann surface

Charles Philip Lanski, Differential identities, Lie ideals, and Posner's theorems

Erich Miersemann, Asymptotic expansion at a corner for the capillary problem

Dietrich W. Paul, Theory of bounded groups and their bounded cohomology

Ibrahim Salama, Topological entropy and recurrence of countable chains

Zbigniew Slodkowski, Pseudoconvex classes of functions. I. Pseudoconcave

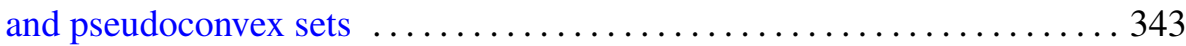

Alfons Van Daele, $K$-theory for graded Banach algebras. II . . . . . . . . . . 377

Edwin Wolf, Functions in $R^{2}(E)$ and points of the fine interior 393 